\title{
Congestion Control of Deregulated Power Systems by Optimal Placement of TCSC using ESMO Algorithm
}

Harish Kumar

Department of Electrical engineering,

University Institute of Engineering \&Technology,

Rohtak,Haryana, India

harishkumarkhola@gmail.com

\section{Ravindra Pratap Singh}

Department of Electrical engineering,

JECRC University,

Iaipur, Rajasthan, India

\begin{abstract}
In the deregulated energy market, congestion represents a fundamental problem for the independent system operator. Moreover, it involves further cost and pretenses a risk to the security of the power system. In this paper, Optimal Power Flow (OPF) and Available Transfer Capability (ATC) based techniques are presented for allocating TCSC to ease the congestion exploiting the Congestion Rent Contribution (CRC) approach on the basis of the position marginal price. In bilateral transactions environment, to calculate the power transfer of dc distribution factors are exploited. The proposed Enhanced Spider Monkey Optimization (ESMO) method is used to deal with congestion in a pool electricity market. The Congestion Management (CM) is examined for contingency and normal because of the line outage and sudden load variation. The effectiveness of the proposed algorithm is examined on IEEE 57-bus as well as 30-bus test systems.
\end{abstract}

Keywords: Congestion; ATC; TCSC; IEEE Bus system; Power flows

\begin{tabular}{ll} 
Nomenclature \\
\hline Abbreviations & Descriptions \\
\hline QOGWO & Quasi-Opposition on the basis of GWO \\
DE & Differential Evolution \\
ORPD & Optimal Reactive Power Dispatch \\
VS & Voltage Stability \\
SVC & Static Var Compensator \\
WOA & Whale Optimization Algorithm \\
LM & Loss Minimization \\
GWO & Grey Wolf Optimization \\
QODE & Quasi-Opposition on the basis of DE \\
BL & Branch Loading \\
FACTS & Flexible Alternating Current Transmission System \\
PSO & Particle Swarm Optimization \\
GA & Genetic Algorithm \\
VCPI & Voltage Collapse Proximity Indication \\
TEP & Transmission Expansion Planning \\
GSA & Gravitational Search Algorithm \\
ITCC & Index Of Total Congestion Cost \\
MG & Microgrid \\
CRO & Chemical Reaction Optimization \\
DLUF & Disparity Line Utilization Factor \\
LL & Local Leader \\
QOCRO & Quasi-Oppositional Chemical Reaction Optimization \\
LLD & Local Leader Decision \\
GLL & Global Leader Learning \\
GL & Global Leader \\
RPI & Real Power Injection \\
ABC & Artificial Bee Colony \\
GLD & Global Leader Decision \\
TLR & Transmission Line Relief \\
TLBO & Teaching Learning Based Optimization \\
ABC & Artificial Bee Colony \\
ACO & Ant Colony Optimization \\
WOA & Whale Optimization Algorithm \\
WG & Wind Generator \\
\hline GResbee Publim & \\
\hline &
\end{tabular}

(C)Resbee Publishers

https://doi.org/10.46253/jcmps.v2i2.a5 


\section{Introduction}

Generally, the power customers and producers are challenging types of electrical energy concoction as well as expenditure in the competitive power market, which might show the way to congestion in a few of the transmission lines in the system. This type of congestion issues symbolizes an often happening occurrence in a restructured power system background that occurs while the transmission line power achieves its highest power flow limit. Moreover, the CM is a method by that to alleviate transmission congestion by maintaining all transactions line and schedules within the limits. The literature study of $\mathrm{CM}$ techniques in that various systems were presented can be established. Using the OPF solution, power production costs are reduced for real power generation scheduling [22]. Moreover, the OPF is needed to deal out the power to optimally convene load demand, whereas controlling all inequality and equality constraints of the system, to extent that the total fuel costs for the generating units are reduced.

To market participants, in the electricity industry, the deregulation has formed new issues. The main technical issue, it has formed in terms of the transmission network is to preserve sufficient ATC to achieve all economic transactions without disconcerting the system. The limitations on the construction of new transmission ability, the cost of creation of extra equipment, social and environmental issues have brought IPP into the electricity market. Additionally, it demands the improvement of ATC of the conventional transmission network. As per the report of NERC, the ATC is considered as the transfer capability measure, which residual in the transmission of the physical network for additional profitable action against and above the already dedicated users. The ATC is estimated on the basis of the ratio of change in power transfer among two buses to the number of transaction achieved in a bus. Moreover, for the bilateral transaction, it has been attained from the AC power flow solution that offers the result of voltage limits, thermal loading, and voltage collapse.

By means of the restricted transmission capability, TCSC has driven out to be the probable way to increase above this restriction [14]. In the system, the lines obtain congested while there is overload. The loading ability of that line needs to increase as well as the system ATC value is enhanced with the recreation of congestion. Installation of TCSC devices alleviated transmission bottlenecks as well as it can enhance voltage stability, power flow controllability, and transient characteristic of the transmission network [18] [19] [20]. Even if the TCSCs installation is extortionate, its price is low in contrast to the installation of storage devices. In a network, to include the TCSCs one must need to determine their optimal positioning and sizing [15] [16] [17].

Various authors [9] [10] [11] [24] [25] [26] have recommended the exploit of sensitivity index based on the performance of the real power index for the optimal positioning of FACTS devices. The technique comprises the determination of the optimal positioning in the TCSCs for CM. On the basis of the performance index of the real power and minimization in total system reactive as well as active power losses, the optimal location is determined. In [12], the optimal location of the IPFC technique was presented in which the load margin was increased. In [13], the optimal location of FACTS controllers has presented it considers Branch Loading, LM, and VS as objectives at once exploiting GA for CM. In [14], two new techniques for the positioning FACTS devices were presented for CM. In general, the main aim of the FACTS device is to determine the positioning that can be either to reduce the total cost of the congestion or to make the best use of social welfare.

In this paper, the two techniques are exploited for $\mathrm{CM}$ in deregulated management. In both the techniques, to allocate TCSC, the CR contribution method is utilized. This method depends on marginal prices, which are power flows and LMP by the lines. The proposed optimization is performed for the reduction of total generation cost and CR in the energy pool market. The rest of the paper is organized as follows: Section 2 describes the literature review; section 3 describes the optimal positioning of TCSC. Section 4 defines the comprehension of OPF using the proposed method. Section 5 describes the results and discussions of the paper and section 6 describes the conclusion of the paper.

\section{Literature Review}

In 2018, Saurav Raj and Biplab Bhattacharyya [1] presented WOA, DE, GWO, QODE, and QOGWO method in order to determine the reactive power planning solution with FACTS devices that was TCSC and SVC. Here, the bus systems such as IEEE 57 and 30 bus test system were exploited for the experimental procedure. Using the power flow analysis approach, the position of TCSC was analyzed and the position of SVC was determined by the VCPI technique. In addition, the aforementioned method was exploited to discover the finest set of all control variables such as the shunt type of FACTS, the series type, TCSC, and SVC devices in the bus system that reduces the system operating cost and active power loss when upholding voltage profile within the permitted limit. 
In 2019, Omid Ziaee et al [2] presented two planning issues into a single planning issue. Moreover, it demonstrates the advantages of the amalgamation of two issues in numerically. In a transmission network, the optimal TEP issue and the optimal position of TCSCs were combined. For the combined issue, when multiple operation scenarios were contemplated, the proposed single period math program reduces the speculation costs of the TCSCs and new lines, as well as the predictable costs of load and generation, shed. By considering the load and wind power generation of a specific period, the operation cases were developed by a proposed technique for the state minimization.

In 2018, Mehdi Abapour et al [3] developed a process to estimate whether exploiting TCSC for transient stability enhancement was lucrative or not. For this process, the transient unsteadiness risk was computed both with and without TCSC. Then, an evaluation was done among the number of enhancement done in speculation cost of TCSC and the transient instability risk. A conditional method was exploited, to compute the transient instability probability.

In 2017, Susanta Duttaa et al [4] presented a competent QOCRO technique to discover the possible optimal result of the multi-objective ORPD issue with FACTS device. To enhance the convergence speed and the solution quality, the quasi-oppositional based learning was included in existing CRO.

In 2017, Rahul Agrawal et al [5] presented approaches for TCSC to determine the optimal position and size of the power system by evolutionary optimization methods namely TLBO, ABC, and PSO. Even though to get maximum benefit, their position and size must be optimal. The reduction of cost of TCSC installation, transmission loss, and voltage deviation was represented as an objective function.

In 2019, Prashant Kumar Tiwari et al [6] developed a simple, well-organized and dependable dualphase optimization scheme to resolve the congestion issue along by means of maximizing the system profit, reduction of congestion cost system generation cost, and emission by optimal positioning \& numbers of TCSC along with wind WGs in a deregulated power market. Moreover, two new indices such as Congestion Detection Index (CDI) and ITCC were presented towards more rapid recognition and minimizations of system congestion efficiently. For this study, the pool and bilateral deregulated market method were represented. Subsequent to optimal positioning of TCSC and WG in the system the maximum system profit was attained.

In 2018, Akanksha Mishra and G.V. Nagesh Kumar [7] presented a DLUF for the optimal positioning and GSA on the basis of the IPFC optimal tuning for CM occurred in the transmission lines. With respect to the relative line congestion, DLUF ranks the transmission lines. Hence, the IPFC was located in the highly and slightly amount of congested line that linked to a similar bus. By GSA the optimal sizing of IPFC was performed. To tune the parameters of the IPFC, a multi-objective function was selected.

In 2017, Ramesh Guguloth and T.K. Sunil Kumar [8] presented a combination of FACTS device and an MG based Congestion Management approach for easing congestion occurs in the transmission line. The optimal positioning of the FACTS device and MG were attained exploiting RPF and TLR sensitivity indices. The FACTS and MG on the basis of the CM come near while combined were establish to be better than the performance while the approaches were performed independently.

\section{Optimal Positioning of TCSC}

\subsection{Modeling of TCSC}

Generally, TCSC is included in a transmission line to help line flows control. Fig 1 shows the basic model of TCSC device, which placed in reactive as well as active power into the bus node where the line is linked. By the injected power, it shows the controller action is simulated. The TCSC evaluation is defined using the reactance and ability of line where it is positioned. 


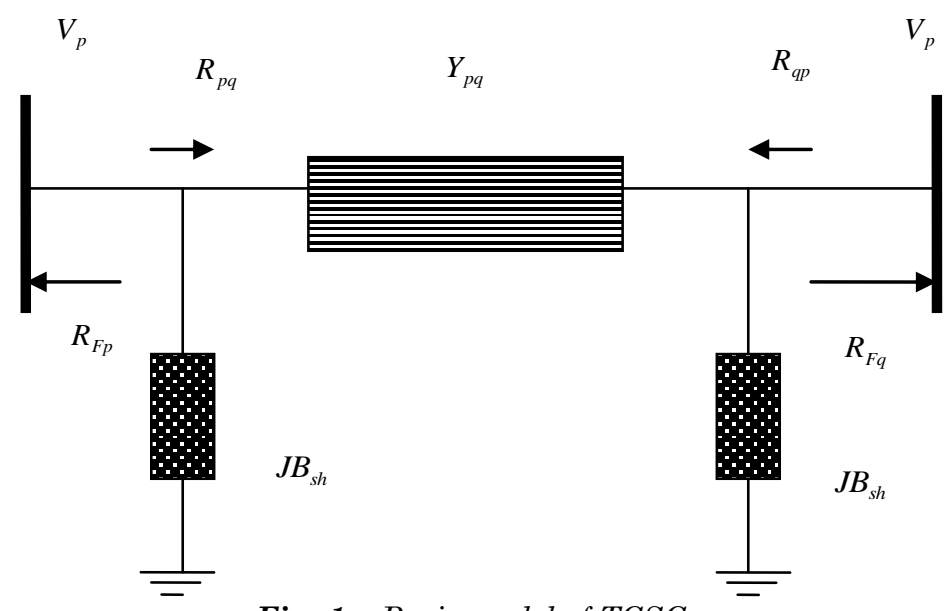

Fig. 1. Basic model of TCSC

$$
\mathrm{Y}_{\mathrm{TCSC}}=\mathrm{D}_{\mathrm{TCSC}} \times \mathrm{Y}_{\mathrm{pq}}
$$

In eq. (1) $Y_{p q}$ indicates the reactance of line among bus $p$ and bus $q$ and $D_{\text {TCSC }}$ represents the compensation degree by TCSC. Subsequent to the TCSC position, the RPI at busp and bus $q$ are stated eq. (2) and (3).

$$
\begin{aligned}
& R_{F, p}=\frac{Y_{T C S C}}{Y_{p, q} \cdot\left(Y_{p, q}-Y_{T C S C}\right)}\left(\theta_{p}-\theta_{q}\right) \\
& R_{F, q}=\frac{Y_{T C S C}}{Y_{p, q} \cdot\left(Y_{p, q}-Y_{T C S C}\right)}\left(\theta_{p}-\theta_{q}\right)
\end{aligned}
$$

While TCSC is positioned in line among bus $p$ and bus $q$, and it modifies the power balance equality constraint and also computed as eq. (4). In eq. (4), $R_{p}$ indicate the net RPI at the bus $p, R_{F p}$ indicates power injection at bus $p$ because of the TCSC installation, $R_{D p}$ represents the power demand at the bus $\mathrm{p}, \mathrm{R}_{\mathrm{Gp}}$ indicates the real power generation at the bus $\mathrm{p}$.

$$
\begin{gathered}
\text { For bus p } R_{p}-R_{G p}+R_{D p}+R_{F, p}=0 \\
\text { For bus q } R_{q}-R_{G q}+R_{D q}+R_{F, q}=0
\end{gathered}
$$

In eq. (5), $R_{F q}$ indicates power injection at bus $q$ because of the TCSC installation, $R_{q}$ indicates net $\mathrm{RPI}$ at the bus $\mathrm{q}, \mathrm{R}_{\mathrm{Gq}}$ indicates active power generation at the bus $q$, and $\mathrm{R}_{\mathrm{Dq}}$ indicates power demand at the bus $q$.

In addition, for OPF formulation, as an inequality constraint, the compensation limit of TCSC is added. Here, the $\mathrm{Y}_{\mathrm{TCSC}}^{\max }$ is taken as $0.5 \cdot \mathrm{Y}_{\mathrm{TCSC}}^{\min }$ is taken as zero and $Y_{p q}$ to avoid overcompensation.

$$
\mathrm{Y}_{\mathrm{TCSC}}^{\min } \leq \mathrm{Y}_{\mathrm{TCSC}} \leq \mathrm{Y}_{\mathrm{TCSC}}^{\max }
$$

\subsection{TCSC Positioning using the CRC Approach}

The position of marginal prices maximizes while the congestion takes place. On the congestion level in that line, the difference of the LMP among two buses offers information. On the other hand, with respect to the low rated line overloading, the difference of the LMP without any help may not be capable to detain the optimal position. On that circumstance, the CRC approach is highly consistent as it comprises the line flows beside the difference of the LMP among buses.

Hence, the difference of the LMP is great but line flow is little, which line is not available in the precedence list. By each line, the list is produced depending on CRC. For TCSC installation, the congested lines are not always the probable position. Therefore, it is necessary to generate a priority list, which limits not only the overloaded lines but also the adjoining lines that are linked to it. The CR is computed as the product of difference of the LMP as well as the line flows stated in eq. (7).

$$
\mathrm{CR}_{\mathrm{pq}}=\Delta \mathrm{LMP}_{\mathrm{p}, \mathrm{q}} \times \mathrm{R}_{\mathrm{p}, \mathrm{q}}
$$

where $\Delta \mathrm{LMP}_{\mathrm{p}, \mathrm{q}}$ represents the difference in marginal prices among bus $\mathrm{p}$ and bus $\mathrm{q}$, and $\mathrm{R}_{\mathrm{pq}}$ represents the line flow among buses $p$ and bus $q$.

Depending on the number of CRC every line obtains ranked in the priority list by individual line and it is stated in eq. (8). 


$$
\mathrm{CRC}=\frac{\mathrm{CR}_{\mathrm{p}, \mathrm{q}}}{\mathrm{TCR}}
$$

where TCR indicates the total CR value and it is stated in eq. (9).

$$
\mathrm{TCR}=\sum_{\mathrm{pq}=1}^{\mathrm{N}_{\mathrm{L}}} \Delta \mathrm{LMP}_{\mathrm{p}, \mathrm{q}} \times \mathrm{R}_{\mathrm{p}, \mathrm{q}}
$$

To consider the optimal position for TCSC, some maximum priority lines are contemplated for TCSC position by $\mathrm{OPF}$ as well as calculating the total $\mathrm{CR}$ and objective function. The optimum position presents the minimum objective function value or minimum $\mathrm{CR}$.

\subsection{ATC Enhancement}

By improving the ATC of the network, the congestion can be managed that can be estimated to exploit sensitivity factors $\mathrm{SF}$. The $\mathrm{SF}_{\mathrm{pq}, \mathrm{ij}}$ is described as the transaction ratio among buses $\mathrm{i}$ and $\mathrm{j}$ flowing by a line linked among buses $\mathrm{p}$ and $\mathrm{q}$. It is denoted in eq. (10).

$$
\mathrm{SF}_{\mathrm{pq}, \mathrm{ij}}=\frac{\mathrm{Y}_{\mathrm{pi}}-\mathrm{Y}_{\mathrm{qi}}-\mathrm{Y}_{\mathrm{pj}}-\mathrm{Y}_{\mathrm{qj}}}{\mathrm{y}_{\mathrm{pq}}}
$$

In eq. (10), $\mathrm{y}_{\mathrm{pq}}$ represents the line, $\mathrm{Y}_{\mathrm{pi}}$ indicates the value of $\mathrm{p}^{\text {th }}$ row and $\mathrm{i}^{\text {th }}$ column of the reactance matrix $Y$.

By the new line flow, the ATC is estimated among buses $\mathrm{p}$ and bus $\mathrm{q}$, due to a bilateral transaction among bus $\mathrm{i}$ and bus j. Eq. (11) represents the new power flow by the line.

$$
R_{p, q}=R_{p, q}^{0}+S_{p q, i j} \times R_{i j}
$$

In eq. (11), $R_{\mathrm{p}, \mathrm{q}}^{0}$ represents the base case power flow by the line and $R_{\mathrm{ij}}$ indicates the transaction amount. Eq. (12) indicates the utmost transferable power without hitting the thermal limit of the line. The ATC is the minimum value of the utmost permissible transactions against all the lines.

$$
\begin{aligned}
\mathrm{R}_{\mathrm{pq}, \mathrm{ij}}^{\max } & =\frac{\mathrm{R}_{\mathrm{pq}}^{\max }-\mathrm{R}_{\mathrm{pq}}^{0}}{\mathrm{SF}_{\mathrm{pq}, \mathrm{ij}}} \\
\mathrm{ATC}_{\mathrm{ij}} & =\min \left(\mathrm{R}_{\mathrm{pq}, \mathrm{ij}}^{\max }\right) \forall \mathrm{pq}
\end{aligned}
$$

\subsection{Formulation of OPF}

The OPF is resolved to estimate the nodal prices as well as power generation to ease congestion in the competitive market. It depends on loads and generator offers while the load is elastic. The objective function can be stated as eq. (14).

$$
\operatorname{Min} \sum_{\mathrm{p}=1}^{\mathrm{q}_{\mathrm{g}}} \mathrm{C}_{\mathrm{p}}\left(\mathrm{R}_{\mathrm{Gp}}\right)-\sum_{\mathrm{q}=1}^{\mathrm{q}_{\mathrm{d}}} \mathrm{B}_{\mathrm{q}}\left(\mathrm{R}_{\mathrm{Gq}}\right)
$$

where $C_{p}\left(R_{G p}\right)$ is the bid curve of $p^{\text {th }}$ generator, $q_{g}$ represents a number of generators, $B_{q} i\left(R_{G q}\right)$ indicates the curve for $q^{\text {th }}$ demand and $q_{d}$ represents the number of loads.

For the inelastic load, whereas the demand of the load does not modify with cost, the objective model turns out to be the generated power total cost which needs to be dispatched when fulfilling the entire system restraints and it is stated in eq. (15).

$$
\operatorname{Min} \sum_{\mathrm{p}=1}^{\mathrm{q}_{\mathrm{g}}} \mathrm{C}_{\mathrm{p}}\left(\mathrm{R}_{\mathrm{Gp}}\right)
$$

\section{Comprehension of OPF using Proposed Method}

\subsection{Conventional Algorithm}

The SMO method is a nature-inspired method [23]. This approach is stochastic in nature; in each step, it initiates few arbitrary modules. The SMO scheme imitates the fusion-fission model of a spider monkey. The main descriptions of the fusion-fission social model are stated as below.

(a) The fusion-fission social model animals endure in a set of 40-50 monkeys, which split the member into subsets in order to search the food to decrease competition. 
(b) Generally, the GL is in charge of exploring the source of food, which guides in the set. These sets are split into little subsets to look for food separately.

(c) The LL guides the subsets and in charge of scheduling an efficient arrangement for foraging route every day.

(d) These set members look for the sources of food and change their location on the basis of the distance from sources of food. In addition, they can able to converse with all set members to sustain a social bond with respect to the stagnation.

The SMO method comprises of six main stages, which is going after by initialization stages. These stages propose that how spider monkey updates their location on the basis of their preceding knowledge and behavior of neighbors.

The first stage is the population initialization. Here, a population of $P$ initialization of spider monkey. Primary population indicated by $\mathrm{N}$-dimensional vector $\mathrm{SN}(\mathrm{i}=1,2, \ldots, \mathrm{P})$. Each $\mathrm{SMO}$ indicates the solution of the crisis optimally under consideration. $S N_{i}$ indicates the spider monkey population and it is shown in eq. (16).

$$
\mathrm{SN}_{\mathrm{ij}}=\mathrm{SN}_{\text {min }}+\mathrm{V}[0,1] \times\left(\mathrm{SN}_{\operatorname{maxj}}-\mathrm{SN}_{\min \mathrm{j}}\right)
$$

In eq. (16), $\mathrm{SN}_{\mathrm{ij}}$ indicates the $\mathrm{i}^{\text {th }}$ source of food in the swarm, $\mathrm{SN}_{\min j}$ represents lower bounds and $\mathrm{SN}_{\min j}$ represents upper bounds of $\mathrm{SN}_{\mathrm{ij}}$ indicates the $\mathrm{j}^{\text {th }}$ direction correspondingly and $\mathrm{V}[0,1]$ is a uniformly distributed arbitrary number in the range $(0,1)$.

The LL is the second stage. Here, it modernizes the position of SMO on the basis of the knowledge of global and local set members. These members evaluate the fitness of the new position and present position and apply greedy selection.

The location $n$ updates for $\mathrm{i}^{\text {th }} \mathrm{SMO}$ of $\mathrm{m}^{\text {th }}$ group is stated in eq (17). Here, $\mathrm{SN}_{\mathrm{ij}}$ denotes the $\mathrm{i}^{\text {th }}$ solution in $\mathrm{j}^{\text {th }}$ dimension, $\mathrm{LL}_{\mathrm{mj}}$ indicates the $\mathrm{j}^{\text {th }}$ dimension of the $\mathrm{m}^{\text {th }}$ local group leader position. $\mathrm{SN}_{\mathrm{rj}}$ denotes the $r^{\text {th }}$ solution that is chosen arbitrarily from $\mathrm{m}^{\text {th }}$ group namely $\mathrm{r} \neq \mathrm{i} . \mathrm{V}[0,1]$ represents a uniformly distributed arbitrary number in the range of $0-1$.

$$
\mathrm{SN}_{\text {newij }}=\mathrm{SN}_{\mathrm{ij}}+\mathrm{V}[0,1] \times\left(\mathrm{LL}_{\mathrm{mj}}-\mathrm{SN}_{\mathrm{ij}}\right)+\mathrm{V}[-1,1] \times\left(\mathrm{SN}_{\mathrm{rj}}-\mathrm{SN}_{\mathrm{ij}}\right)
$$

In GL stage, it initiates after concluding the local leader stage. Here, the location gets updated on the basis of the previous knowledge of the global group members and local Leader with the assist of eq. (18).

$$
\mathrm{SN}_{\text {newij }}=\mathrm{SN}_{\mathrm{ij}}+\mathrm{V}[0,1] \times\left(\mathrm{GL}_{\mathrm{j}}-\mathrm{SN}_{\mathrm{ij}}\right)+\mathrm{V}[-1,1] \times\left(\mathrm{SN}_{\mathrm{rj}}-\mathrm{SN}_{\mathrm{ij}}\right)
$$

where $\mathrm{GL}_{\mathrm{j}}$ communicate to the $\mathrm{j}^{\text {th }}$ dimension of the global leader position as well as $\mathrm{j} \in\{1,2, \ldots \mathrm{N}\}$ is arbitrarily chosen within the dimension. Here, the $\mathrm{SN}_{\mathrm{j}}$ updates their location, which is on the basis of their probabilities $\left(\right.$ prob $_{\mathrm{i}}$ ) that are computed by their fitness. There can be various techniques for probability computation although it must be a function of fitness. The fitness function represents regarding its fitness quality is represented in eq. (19), computation be obliged to comprise function value.

$$
\left(\text { prob }_{\mathrm{i}}\right)=0.9 \times \frac{\text { fit }_{\mathrm{i}}}{\text { fit }_{\max }}+0.1
$$

In the GLL stage, SMO alters the location of a global leader with the assist of greedy methods. In a current swarm, the highly fitted solution is selected as a global leader. Additionally, it carried out to confirm the location of GL is modernized or not and modify Global Limit Count. At present in LLL stage position of local leader is modified with the assist of greedy methods. The extremely fitting solution in the current swarm is selected as LL. Additionally, it achieves a check that the position of LL is modernized or not and adapts Local Limit Count.

In the LLD stage, the decision is taken regarding the location of LL, if it is not modernized up to a threshold also represented as LL Limit $\left(\mathrm{LL}_{\text {limit }}\right)$. If there is no alter it arbitrarily initializes location of LL. Location of LL might be decided using eq. (20).

$$
\mathrm{SN}_{\text {newij }}=\mathrm{SN}_{\mathrm{ij}}+\mathrm{V}[0,1] \times\left(\mathrm{GL}_{\mathrm{j}}-\mathrm{SN}_{\mathrm{ij}}\right)+\mathrm{V}[0,1] \times\left(\mathrm{SN}_{\mathrm{rj}}-\mathrm{LL}_{\mathrm{mj}}\right)
$$

From eq. (20) the updated dimension of this SM is attracted toward a GL and keeps away from the LL.

GLD stage takes the decision regarding the location of GL if it is not modernized up to a threshold is called as GL Limit $\left(\mathrm{GL}_{\lim i t}\right)$, and subsequently, GLD produces subsets of little size. In this stage, LLs are produced for new subsets exploiting LL Learning procedure. 


\subsection{Proposed Enhanced SMO (ESMO) Algorithm}

The Spider Monkey is the most recent method in the different swarm intelligence field. The proposed ESMO method enhances the fundamental SMO method performance. The ESMO recommended various enhancements in the LL stage of fundamental SMO. Location update equation in proposed ESMO obtains an average difference between the present and arbitrarily produced locations. It creates an arbitrary location in a given range for a particular issue. This recommended modification accelerates the convergence rate and increases consistency. Moreover, it is supposed that enhanced fitted solution has the best solution in their immediacy.

$$
\mathrm{X}_{\mathrm{ij}}=\mathrm{Y}_{\mathrm{ij}}+\varphi_{\mathrm{ij}} \times\left(\mathrm{LL}_{\mathrm{mj}}-\mathrm{ESN}_{\mathrm{ij}}\right)+\varphi_{\mathrm{ij}} \times\left(\frac{\mathrm{sum}}{\mathrm{SN}}\right)
$$

where $\varphi_{\mathrm{ij}}$ is a uniformly produced arbitrary number in the range $(0,1)$.

Where $\mathrm{ESN}_{\mathrm{ij}}$ indicates the $\mathrm{j}^{\text {th }}$ dimension of the $\mathrm{i}^{\text {th }} \mathrm{ESMO}, \mathrm{LL}_{\mathrm{mj}}$ make sure the $\mathrm{j}^{\text {th }}$ dimension of the $\mathrm{m}^{\text {th }}$ local leader set position. The SN indicates the source of food, which is arbitrarily produced using the location for a source of food. SUM is the difference average for current location and arbitrarily created a location. Eq. (21) updates highly fitted solutions by inspiration from optimal Swarm Intelligence.

This new addition in conventional SMO maximizes the balance among exploitation and exploration of general possible solutions.

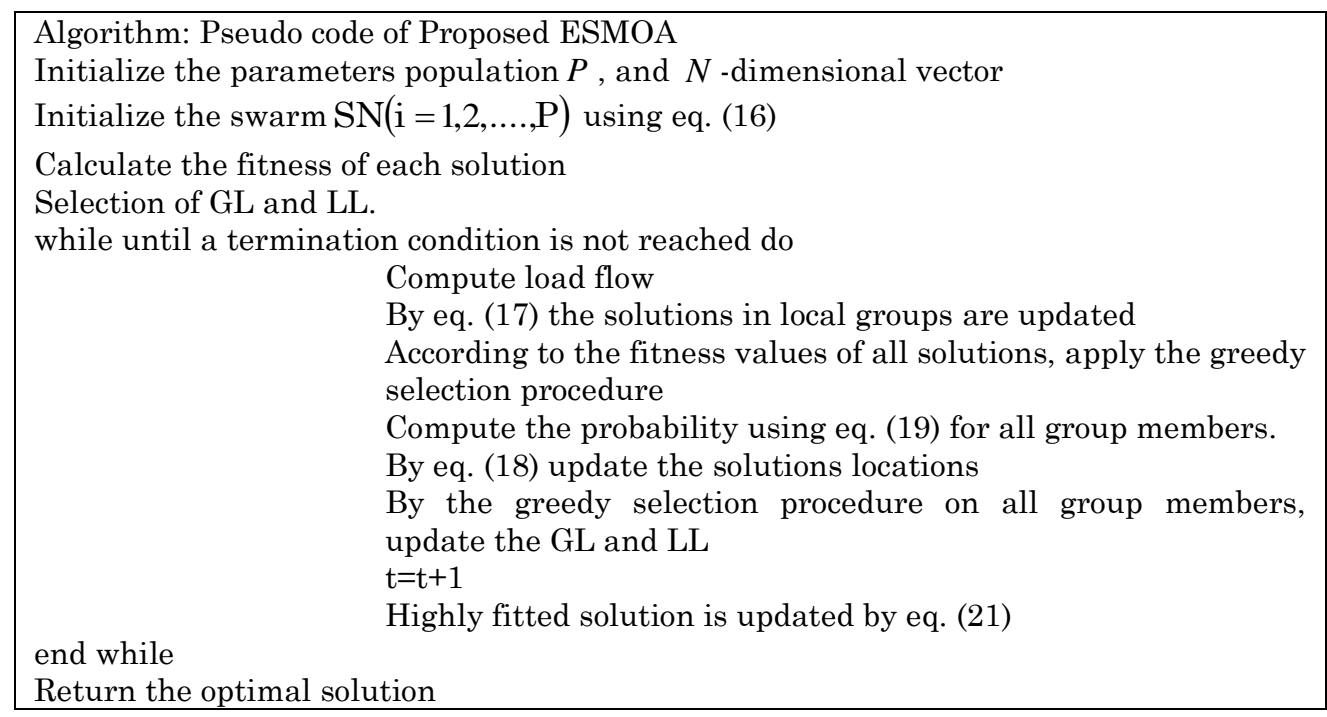

\section{Results and Discussions}

\subsection{Simulation Setup}

The OPF problem is resolved by a proposed ESMOA technique. The experimentation was performed for various contingency scenarios and optimum TCSC allocation. Three contingency Cases, are 1, 2, and 3, were analyzed for line outage, maximize in load at buses, and minimization in the capacity of the line and its TCR is $88.55,143.56$ and 65.46 .

\subsection{IEEE 30 Bus System}

In Tables 1, the performance analysis of the proposed and conventional methods is shown for TCSC allocation. The first three lines for TCSC allocation are exploited to get rid of congestion and attain the OPF solution. It is observed from Table 1 that the proposed ESMO on the basis of the OPF recommends a superior solution and yields an important minimization in generation cost under different cases.

In fig 2, the graphical representation of TCR obtained using proposed and conventional ABC, WOA and, ACO algorithm for all three cases such as 1, 2, and 3 in the IEEE 30-bus test system, are demonstrated. 


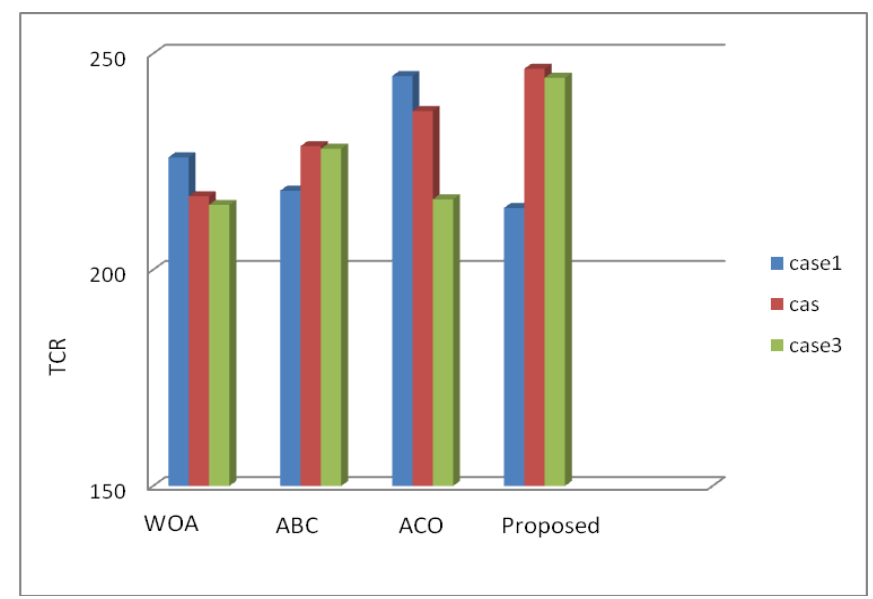

Fig. 2. Performance analysis of proposed and existing systems on IEEE 57 test bus system for TCR (\$/hr)

Table 1. Performance Analysis of OPF with TCSC allocation for IEEE 30 Bus Test System

\begin{tabular}{llllllllll}
\hline Cases & $\mathbf{1}$ & \multicolumn{3}{c}{$\mathbf{2}$} & \multicolumn{5}{c}{ 3 } \\
TCSC place & line 9 & line 2 & line 1 & line 7 & line 2 & line 4 & line 6 & line 9 & line 9 \\
WOA & 214.23 & 222.32 & 223.45 & 323.24 & 314.23 & 312.32 & 223.45 & 223.14 & 214.83 \\
ABC & 212.34 & 214.32 & 132.23 & 211.23 & 312.34 & 314.32 & 232.23 & 211.13 & 312.44 \\
ACO & 132.34 & 212.12 & 211.23 & 310.12 & 232.34 & 212.12 & 211.13 & 210.22 & 232.69 \\
Proposed & 114.03 & 112.92 & 123.65 & 223.21 & 214.27 & 202.12 & 203.10 & 103.74 & 204.13 \\
\hline
\end{tabular}

\subsection{IEEE 57 Bus System}

From Tables 2, it is seen that the TCSC integration outcomes in minimization in the generation cost in all the contingency scenarios. This shows the effectiveness of generation cost method for the allocation of TCSC to deal with congestion in the network. The overall result analysis shows that the proposed technique is superior to the conventional WOA, ABC and ACO methods.

The graphical representation of TCR attained by conventional methods and the proposed ESMOA approach for all three scenarios such as 1, 2, and 3 in the IEEE 57-bus test system is shown in Fig. 3.

Table 2. Performance Analysis of OPF with allocation of TCSC for IEEE 57 Bus Test System

\begin{tabular}{crrrrrrrrrr}
\hline Cases & \multicolumn{3}{c}{ 1A } & \multicolumn{3}{c}{ 2A } & \multicolumn{3}{c}{ 3A } \\
\hline TCSC place & line 9 & line 2 & line 1 & line 7 & line 2 & line 4 & line 6 & line 9 & line 9 \\
WOA & 221.32 & 221.23 & 322.33 & 112.24 & 123.24 & 321.32 & 145.23 & 322.33 & 182.24 \\
ABC & 222.34 & 312.45 & 318.34 & 132.23 & 112.23 & 222.34 & 212.45 & 318.34 & 192.23 \\
ACO & 214.56 & 220.23 & 315.26 & 102.45 & 122.01 & 324.56 & 140.23 & 315.26 & 112.45 \\
Proposed & 121.92 & 211.83 & 302.13 & 101.04 & 103.34 & 221.12 & 101.29 & 222.33 & 102.24 \\
\hline
\end{tabular}

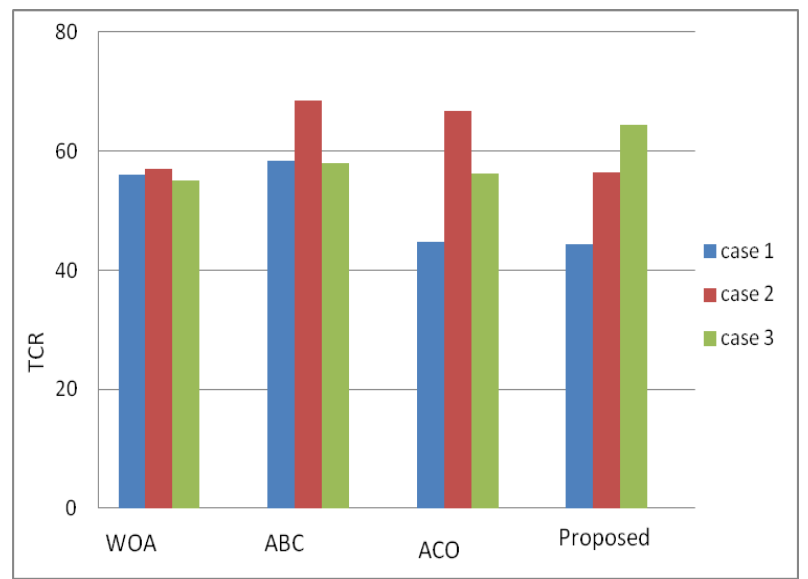

Fig. 3. Performance analysis of proposed and existing systems on IEEE 57 test bus system for TCR (\$/hr)

\section{Conclusion}

In this paper, to control congestion, two techniques were used in a deregulated environment such as $\mathrm{OPF}$ based approach and ATC based approach. In both the techniques, the allocation of TCSC was performed 
exploiting CR approach on the basis of the location marginal prices. The proposed ESMO was exploited as an optimization approach to reduce the total cost of generation or CR in pool-based energy market for OPF. The OPF solutions attained by WOA, ABC, and ACO based approaches are compared. Finally, the outcomes demonstrate that the proposed method has faster convergence and it tends to least amount of CR and important savings in the cost of generation. For bilateral transactions, the ATC based approach exploits linear sensitivity factors SFs to calculate ATC by DC power flow approach. Enhancement of ATC for congestion control was performed by reducing the CR by TCSC position. Both contingency and normal circumstances were analyzed for CM. Finally, the results revealed that CR contribution method was competent in allocating TCSC to control congestion. In addition, the OPF technique shows potential than the ATC technique as it obtains the cost of generation also into deliberation that optimally allocates the TCSC device for CM in a deregulated environment.

\section{Compliance with Ethical Standards}

Conflicts of interest: Authors declared that they have no conflict of interest.

Human participants: The conducted research follows the ethical standards and the authors ensured that they have not conducted any studies with human participants or animals.

\section{References}

[1] Saurav Raj, Biplab Bhattacharyya, "Optimal placement of TCSC and SVC for reactive power planning using Whale optimization algorithm", Swarm and Evolutionary Computation, vol. 40, pp. 131-143, June 2018.

[2] O. Ziaee, O. Alizadeh-Mousavi and F. F. Choobineh, "Co-Optimization of Transmission Expansion Planning and TCSC Placement Considering the Correlation Between Wind and Demand Scenarios," IEEE Transactions on Power Systems, vol. 33, no. 1, pp. 206-215, Jan. 2018.

[3] M. Abapour, P. Aliasghari and M. Haghifam, "Risk-based placement of TCSC for transient stability enhancement," in IET Generation, Transmission \& Distribution, vol. 10, no. 13, pp. 3296-3303, 6102016.

[4] Susanta Dutta, Sourav Paul, Provas Kumar Roy,"Optimal allocation of SVC and TCSC using quasi-oppositional chemical reaction optimization for solving multi-objective ORPD problem", Journal of Electrical Systems and Information Technology, vol. 5, no. 1, pp. 83-98, May 2018.

[5] Rahul Agrawal, S. K. Bharadwaj, D. P. Kothari, "Population based evolutionary optimization techniques for optimal allocation and sizing of Thyristor Controlled Series Capacitor", Journal of Electrical Systems and Information Technology, vol. 5, no. 3, pp. 484-501, December 2018.

[6] Prashant Kumar Tiwari, Manash Kumar Mishra, Subhojit Dawn, "A two step approach for improvement of economic profit and emission with congestion management in hybrid competitive power market", International Journal of Electrical Power \& Energy Systems, vol. 110, pp. 548-564,September 2019.

[7] Akanksha Mishra, Venkata Nagesh Kumar G,"Congestion management of deregulated power systems by optimal setting of Interline Power Flow Controller using Gravitational Search algorithm", Journal of Electrical Systems and Information Technology, vol. 4, no. 1, pp. 198-212, May 2017.

[8] Ramesh Guguloth, T. K. Sunil Kumar,"Congestion management in restructured power systems for smart cities in India", Computers \& Electrical Engineering, vol. 65, pp. 79-89, January 2018.

[9] S.N. Singh, A.K. David, "Optimal location of FACTS devices for congestion management", Electric Power Syst. Res. 58, 71-79, June 2001.

[10] H. Besharat, and S.A. Taher, "Congestion management by determining optimal location of TCSC in deregulated power systems", Electrical Power and Energy Systems 30, 563-568, June 2008.

[11] M. Mandala, and C.P. Gupta, "Congestion management by optimal placement of FACTS device", PEDES and Power India, 2010,pp. 1-7, New Delhi, India

[12] R. Minguez, F.Milano, R Zarate-Minano, A.J. Conejo,"Optimal Network Placement of SVC Devices", IEEE Trans. On Power Systems, Vol.22, No.4, Nov.2007.

[13] S.S. Reddy, M.S.Kumari and M. Sydulu, "Congestion management in deregulated power system by optimal choice and allocation of FACTS controllers using multi-objective genetic algorithm ", Transmission and Distribution Conference and Exposition,IEEE PES,pp. 1-7, April 2010.

[14] N. Acharya, N. Mithulananthan, "Locating series FACTS devices for congestion management in deregulated electricity markets", Electric Power Systems Research, Volume 77, Issues 3-4, March 2007, Pages 352-360.

[15] O. Ziaee and F. F. Choobineh, "Optimal location-allocation of TCSC devices on a transmission network," IEEE Transactions on Power Systems, vol. 32, no. 1, pp. 94-102, Jan 2017.

[16] G. Y. Yang, G. Hovland, R. Majumder, and Z.-Y. Dong, "TCSC allocation based on line flow based equations via mixed-integer programming," Power Systems, IEEE Transactions on, vol. 22, no. 4, pp. 2262-2269, Nov 2007.

[17] O. Ziaee and F. Choobineh, "Thyristor-controlled switch capacitor placement in large-scale power systems via mixed integer linear programming and Taylor series expansion," in PES General Meeting Conference Exposition, 2014 IEEE, July 2014, pp. 1-5.

[18] S. Singh and A. David, "Optimal location of FACTS devices for congestion management," Electric Power Systems Research, vol. 58, no. 2, pp. $71-79,2001$. 
[19] F. Alhasawi and J. Milanovic, "Techno-economic contribution of FACTS devices to the operation of power systems with high level of wind power integration," Power Systems, IEEE Transactions on, vol. 27, no. 3, pp. 1414-1421, Aug 2012.

[20] J. Taylor and F. Hover, "Linear relaxations for transmission system planning," Power Systems, IEEE Transactions on, vol. 26, no. 4, pp. 2533-2538, Nov 2011.

[21] D. Lauria, G. Mazzanti, and S. Quaia, "The loadability of overhead transmission lines part i: Analysis of single circuits," IEEE Transactions on Power Delivery, vol. 29, no. 1, pp. 29-37, Feb 2014.

[22] Guguloth Ramesh , Kumar TKSunil, "Optimal power flow based congestion management in deregulated power systems by classical and intelligence methods", Int J Power Energy Syst;vol.36 no. (3), pp:104-11, 2016.

[23] Bansal, Jagdish , Sharma, Harish , Jadon, Shimpi and clerc, maurice, "Spider Monkey Optimization algorithm for numerical optimization. Memetic Computing", 2014.

[24] SB Vinay Kumar, PV Rao, Manoj Kumar Singh,"Multi-culture diversity based self adaptive particle swarm optimization for optimal floorplanning",Multiagent and Grid Systems, vol14, no.1, pp.31-65, 2018.

[25] R Gupta Roy, D Baidya,"Speed Control of DC Motor Using Fuzzy-Based Intelligent Model Reference Adaptive Control Scheme",Advances in Communication, Devices and Networking, Lecture Notes in Electrical Engineering book series, Springer, vol. 462, pp.729-735, 2018.

[26] G Singh, VK Jain, A Singh, "Adaptive network architecture and firefly algorithm for biogas heating model aided by photovoltaic thermal greenhouse system",Energy \& Environment, vol. 29 (7), pp.1073-1097,2018. 